\title{
Backscattering by and Propagation Through the Melting Layer of Precipitation: A New Polarimetric Model
}

\author{
H. W. J. Russchenberg and L. P. Ligthart, Senior Member, IEEE
}

\begin{abstract}
A simple physical model of the melting layer of precipitation is presented. It is able to simulate the polarizationdependence of radar reflections and radio signals propagating through the melting layer. The radar observables are calculated in the Rayleigh regime. The propagation observables are given in the range of 5-50 GHz, and are calculated with an extended Rayleigh approximation. The model requires the rain intensity and the mass density of the snowflakes as input parameters. Radar observations, made with the Delft Atmospheric Research Radar, are used to discuss physical processes in the melting layer. Finally, model simulations are compared with radar data obtained with the Chilbolton radar of the Rutherford Appleton Laboratories.
\end{abstract}

\section{INTRODUCTION}

I N STRATIFORM precipitation, raindrops result from melting snowflakes. On their way down in rain-bearing clouds, dry snowflakes pass the $0^{\circ} \mathrm{C}$ level and start to melt. The region in which they melt is called the melting layer, or, referring to the enhanced radar reflectivity it causes, the bright band. With the growing use of high frequencies for satellite communication links and with the advent of spacebased rain and cloud radars, knowledge of the influence of the melting layer becomes increasingly important. In this paper a new radar-derived physical model of the melting layer is used to simulate height profiles of Doppler and polarimetric radar observations, as well as co- and cross-polar propagation characteristics of satellite signals. The model has two input parameters: the rain intensity and the mass density of the snowflakes before melting. The latter is important to simulate significantly different radar measurements.

The model is developed in the context of propagation research with the Olympus satellite of the European Space Agency, as was coordinated by the Olympus Propagation Experimenters Group OPEX. Within OPEX a special working group was dedicated to the application of radar to propagation modeling. The study of the melting layer model was one the key topics in this working group [1]. Various studies, e.g., [2], [3], have revealed detailed and complex microphysical processes in the melting layer, but radar experiments showed

Manuscript received November 2, 1993; revised May 18, 1995. This work was supported in part by the European Space Agency.

The authors are with the Department of Electrical Engineering, Delft University of Technology, 2600 GA Delft, The Netherlands.

Publisher Item Identifier S 0196-2892(96)00298-7. a response of the melting layer to radar waves that was comparable during different events [4]. This gave rise to the idea that some simple fundamental processes must govern the behavior of the melting layer, and that a model of the melting layer could do without many details of microphysical properties. In this paper, radar measurements of the melting layer are shown and discussed. Based on these measurements, the model is developed and compared with data from another radar. The model is used to predict the influence of the melting layer on a $30-\mathrm{GHz}$ satellite link, being one of the frequencies of the Olympus propagation payload.

\section{THE RADAR OBSERVABLES}

During melting the fall speed $v\left(D_{m s}\right)$ of the particles with diameter $D_{m s}$ changes. The radar, however, observes a reflectivity-weighted fall speed $V_{d}$, given as

$$
\begin{aligned}
V_{d} & =\frac{\int v\left(D_{m s}\right) \sigma_{m s}\left(D_{m s}\right) d D_{m s}}{\sigma_{m s}^{\text {tot }}} \\
& =\frac{\int v\left(D_{m s}\right) N_{m s}\left(D_{m s}\right) D_{m s}^{6} d D_{m s}}{\int N_{m s}\left(D_{m s}\right) D_{m s}^{6} d D_{m s}}
\end{aligned}
$$

in which $N_{m s}\left(D_{m s}\right)$ is the distribution of particle sizes, $\sigma_{m s}\left(D_{m s}\right)$ is the radar cross-section of a melting snowflake, and $\sigma_{m s}^{\text {tot }}$ is the radar cross-section of a radarvolume filled with melting snowflakes. Polarimetric measurements are expressed by several radar observables. The most familiar is the horizontal reflectivity factor $Z_{h h}$, which is copolarly measured with horizontal polarization. The polarization dependence of the radar signal is described by the differential reflectivity $Z_{d r}$, and the linear depolarization ratio $L_{d r}$ :

$$
\begin{aligned}
Z_{h h}= & \frac{\lambda^{4}}{\pi^{5}\left|K_{r}\right|^{2}} \\
& \cdot \int \sigma_{h h}\left(D_{m s}, \Omega\right) N_{m s}\left(D_{m s}\right) t(\Omega) d D_{m s} d \Omega \\
Z_{d r}= & \frac{Z_{h h}}{Z_{v v}} \\
= & \frac{\int \sigma_{h h}\left(D_{m s}, \Omega\right) N_{m s}\left(D_{m s}\right) t(\Omega) d D_{m s} d \Omega}{\int \sigma_{v v}\left(D_{m s}, \Omega\right) N_{m s}\left(D_{m s}\right) t(\Omega) d D_{m s} d \Omega}
\end{aligned}
$$




$$
\begin{aligned}
L_{d r} & =\frac{Z_{h v}}{Z_{v v}} \\
& =\frac{\int \sigma_{h v}\left(D_{m s}, \Omega\right) N_{m s}\left(D_{m s}\right) t(\Omega) d D_{m s} d \Omega}{\int \sigma_{v v}\left(D_{m s}, \Omega\right) N_{m s}\left(D_{m s}\right) t(\Omega) d D_{m s} d \Omega}
\end{aligned}
$$

with $\sigma_{k l}$ as the radar cross-section measured with transmitted polarization $k$ and received polarization $l$, and $t(\Omega)$ as the distribution of solid angles of particle orientation. The radar wavelength is denoted by $\lambda . K_{r}$ is factor that depends on the permittivity of the raindrops. The radar does not measure a reflectivity factor, but a radar cross-section. The conversion of the latter into the former is done with the assumption of Rayleigh-scattering by raindrops. When other forms of precipitation are measured, the equivalent reflectivity factor is calculated as if the scattering was caused by rain. Whenever in this paper reference is made to reflectivity, the equivalent reflectivity factor is meant.

Polarimetric observations of rainfall are optimally performed with the radar pointed toward the horizon. For fallspeed analyses, however, Doppler measurements need to be done with the radar pointed toward the zenith. To combine the two, the radar elevation has to be set to a position in between.

\section{THE PROPAGATION PARAMETERS}

An electric field $E_{t} \exp \left(j \phi_{t}\right)$ that enters the melting layer will undergo attenuation, phase shift, and cross-polarization: it will be seen by the receiver as $E_{t r} \exp \left(j \phi_{t r}\right)$, with $t$ and $r$ denoting the polarization of the transmitter and receiver. The following definitions apply:

$$
\begin{aligned}
& \text { the copolar attenuation } C P A=\frac{E_{h h}^{2}}{E_{t}^{2}} \\
& \text { the cross-polar discrimination } X P D=\frac{E_{h v}^{2}}{E_{h h}^{2}} \\
& \text { the differential attenuation } C P D=\frac{E_{h h}^{2}}{E_{v v}^{2}} \\
& \text { the copolar phase shift } \phi_{h h} \\
& \text { the copolar differential phase shift } \phi_{h h}-\phi_{v v} \\
& \text { the cross-polar differential phase shift } \phi_{h h}-\phi_{h v} .
\end{aligned}
$$

A useful way of presenting the propagation characteristics of the melting layer is by comparing the range-integrated effect of it with the effect a layer with the same thickness, but uniformly filled with rain of the intensity as just below the melting layer, would have. It is quantified by the excess parameters

$$
\begin{aligned}
e C P A= & C P A_{m l}[\mathrm{~dB}]-C P A_{r}[\mathrm{~dB}] \\
e C P D= & C P D_{m l}[\mathrm{~dB}]-C P D_{r}[\mathrm{~dB}] \\
e X P D= & X P D_{m l}[\mathrm{~dB}]-X P D_{r}[\mathrm{~dB}] \\
& e \phi_{h h} \\
& e\left(\phi_{h h}-\phi_{v v}\right) \\
& e\left(\phi_{h h}-\phi_{h v}\right)
\end{aligned}
$$

TABLE I

RELEYANT EQUTPMENT PARAMETERS OF THE Radars as They ARE Used IN THIS Study

\begin{tabular}{|l|l|l|}
\hline & Delft & Chilbolton \\
\hline Frequency [GHz] & 3.315 & 3.075 \\
\hline Elevation & $30^{\circ}$ & $0^{\circ}$ \\
\hline Beamwidth & $1.8^{\circ}$ & $0.25^{\circ}$ \\
\hline Range resolution [m] & 150 & 150 \\
\hline
\end{tabular}

Differential reflectivity Zdr [dB], Mean Doppler speed Vd [m/s]

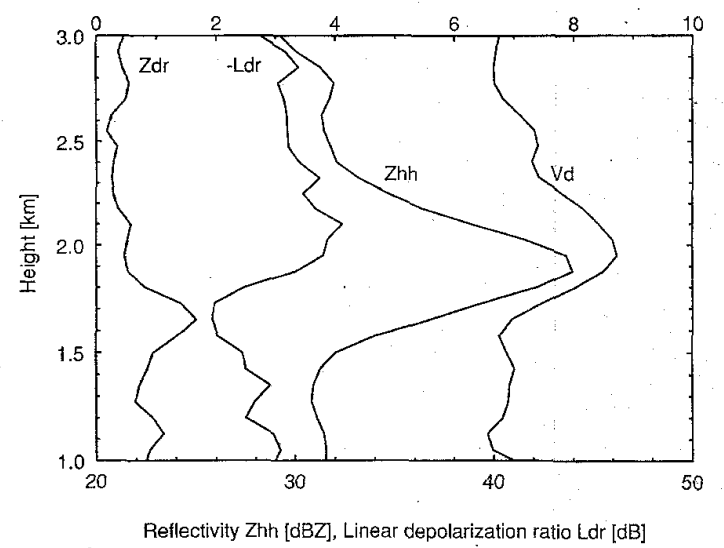

Fig. 1. Measured height profile of the radar observables in the melting layer, measured with the Delft radar. Moderate rain intensity. See Table I for details.

in which the subscripts $m l$ and $r$ refer to the melting layer and rain, respectively. These propagation characteristics are calculated with the extended Rayleigh approximation [5] of forward scattering by spheroids at frequencies up to $50 \mathrm{GHz}$.

\section{Radar Measurements of the Melting Layer}

In this section radar measurements of the melting layer, done with the Delft radar, are discussed; details of the experiment are given in Table I. Fig. 1 shows a typical height profile of $Z_{h h}, Z_{d r}, L_{d r}$, and $V_{d}$ in the melting layer. The data is integrated over $32 \mathrm{~s}$ and $300 \mathrm{~m}$. The $0^{\circ} \mathrm{C}$ isotherm was located at an altitude of approximately $2200 \mathrm{~m}$. From the onset of melting, $Z_{h h}$ increases until it reaches a peak of approximately $15 \mathrm{~dB}$ relative to its value before melting. Then it decreases again to a, more or less, constant value in the rain region underneath the melting layer.

When a snowflake melts, its liquid water content increases. The mass density increases and the particle becomes smaller. It experiences less air resistance then, which causes the fall speed to increase. Assuming stationarity, the number concentration of melting particles decreases. The increase of the water content leads to a larger permittivity of the particle, which causes an increase of the radar reflectivity. Due to the decreasing number concentration, the radar reflectivity decreases again in the lower portion of the melting layer. As a result, the melting layer causes a band of enhanced reflectivity: the bright band. These relationships have long been known in general form, e.g., [6], but details of the melting process and its effect on radar profiles have been difficult to determine. 


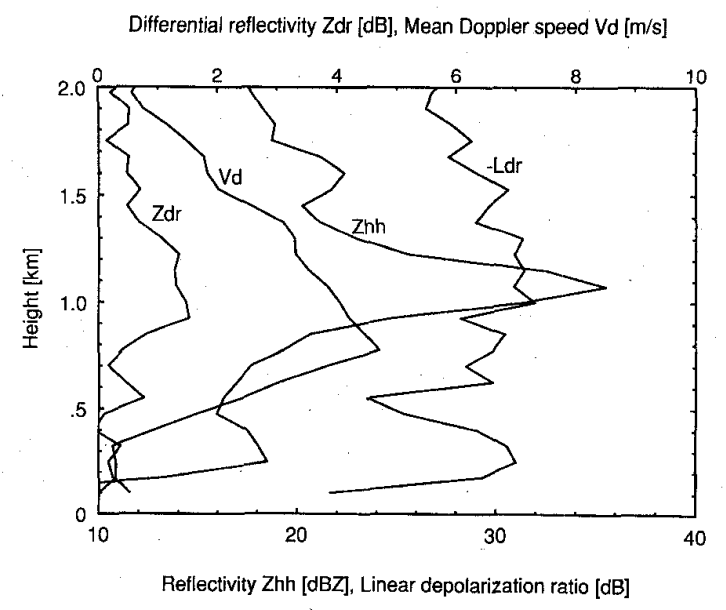

Fig. 2. Measured height profile of the radar observables in the melting layer, measured with the Delft radar. Low rain intensity. See Table I for details.

The height profile of $Z_{d r}$ is somewhat different than the one of $Z_{h h} . Z_{d r}$ increases as well during melting, but its maximum is shifted with respect to the peak of $Z_{h h}$. $Z_{d r}$ shows a sudden peak of approximately $1 \mathrm{~dB}$ with respect to its value in the snow region. Underneath the melting layer $Z_{d r}$ is more or less stable around $0.5 \mathrm{~dB}$. $Z_{d r}$ is sensitive to the shape and orientation of the reflecting particles. The peak value of $Z_{d r}$ at the bottom of the melting layer indicates an oblate mean shape at that height. The small $Z_{d r}$ above and in the upper part of the melting layer indicates a spherical mean shape. However, this does not necessarily imply that melting snowflakes are spherical, but rather that the ensembleaveraged shape is spherical. The individual snowflakes can be very irregular, randomly oriented, structures.

The height profile of $L_{d r}$ is similar to that of $Z_{d r}$. However, high resolution radar measurements with the Chilbolton radar, shown in Section VIII, showed a larger $L_{d r}$ peak and also that the $Z_{d r}$ and $L_{d r}$ peaks not always coincide: the $L_{d r}$ peak is found above the $Z_{d r}$ peak then. Measurements with the Delft Atmospheric Research Radar are done with less resolution, and are therefore less sensitive to this shift, although it has been observed occasionally.

The measured height profile of $V_{d}$ (positive when directed toward the radar) is not representative for the hydrometeors involved. It is mainly caused by wind, because the measurement was done with a $30^{\circ}$ elevation angle.

The just discussed event is one with a moderate rain intensity. The radar observables can be somewhat different in events with a low rain intensity and weak bright band. Fig. 2 depicts such a situation. The radar observables were obtained during the passage of a cold front. The $0^{\circ} \mathrm{C}$ isotherm was located at approximately $1200 \mathrm{~m}$, and the ground temperature was approximately $8^{\circ} \mathrm{C}$. The peak value of $Z_{h}$ is approximately 12 $\mathrm{dB}$ relative to the value of $Z_{h h}$ underneath the melting layer. However, the absolute level of $Z_{h h}$ is approximately $13 \mathrm{~dB}$ lower than in the firstly discussed event. $Z_{d r}$ starts to increase at the onset of melting, rather than halfway through the melting layer as was the case in Fig. 1. Also, the peak value of $Z_{d r}$

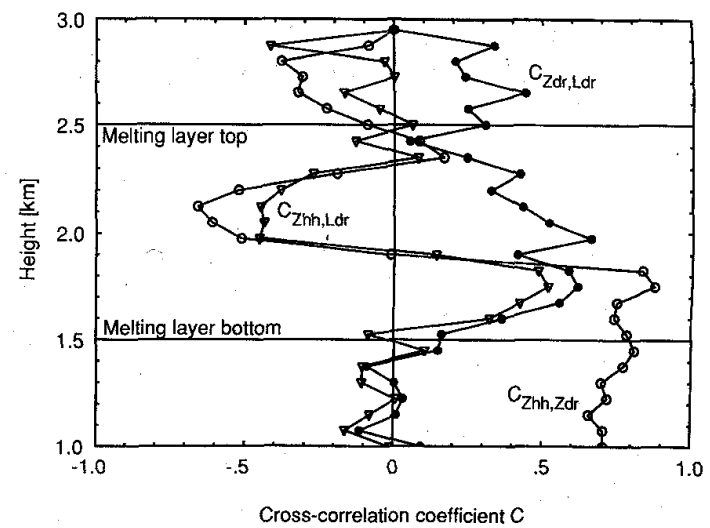

Fig. 3. Height profile of the cross-correlation coefficients of the polarimetric radar observables, during the event of Fig. 1 . The individual observables were integrated over $300 \mathrm{~m}$ and $32 \mathrm{~s}$. The correlation coefficients were calculated with $42 \mathrm{~min}$ worth of data.

is larger than in Fig. 1. $L_{d r}$ is small in the melting layer and shows a peak at the bottom.

\section{The Correlation Between the RadAR ObSERVABLes}

The correlation-coefficient of different radar observables contains information about microphysical phenomena. For instance, when during an event the orientation of the particles becomes more random, $Z_{d r}$ decreases and $L_{d r}$ increases. The cross-correlation coefficient of the $Z_{d r}$ and $L_{d r}$ values of such an event would be negative. Another example: when the particle size increases, $Z_{h h}, Z_{d r}$, and $L_{d r}$ increase as well: the correlation-coefficient will be positive. Such an exercise has been applied to the two events, described in Section IV. The cross-correlation coefficient between the polarimetric radar observables is calculated for each range bin in the melting layer. The individual observables are integrated over $32 \mathrm{~s}$ and $300 \mathrm{~m}$. A rigorous discussion of the correlation analysis of radar data is given in [4]. The correlation analysis will be used in Section VI as a starting point for modeling the size-axial ratio relationship.

Fig. 3 shows the height dependence of the cross-correlation coefficients of the polarimetric radar observables in the melting layer, measured during the event of Fig. 1; the total observation time was $42 \min . Z_{h h}$ is negatively correlated to $Z_{d r}$ and $L_{d r}$ in the upper half of the melting layer. This means that when $Z_{h h}$ increases, $Z_{d r}$ and $L_{d r}$ decrease, or vice versa. In the lower half of the melting layer, $Z_{h h}$ is positively correlated to $Z_{d r}$ and $L_{d r}$ : when $Z_{h h}$ increases, $Z_{d r}$ and $L_{d r}$ increase as well. Finally, $Z_{d r}$ and $L_{d r}$ are positively correlated throughout the whole melting layer.

$Z_{h h}$ is sensitive to particle size while $Z_{d r}$ and $L_{d r}$ are sensitive to particle shape and orientation. When, as in the case of rain, large particles are more oblate than small ones, then $Z_{h h}$ would be positively correlated to $Z_{d r}$ and $L_{d r}$ : they will all increase when the particle size, and consequently, the oblateness increases. However, in the upper half of the melting layer a negative correlation is observed. In general, a negative correlation between $Z_{h h}$ and $L_{d r}$ could be caused by variations 
of particle orientation, because then $Z_{h h}$ decreases slightly, whereas $L_{d r}$ increases. However, $Z_{d r}$ decreases then as well, which has to result in a negative correlation between $Z_{d r}$ and $L_{d r}$. This is not observed.

The negative correlation may be explained by assuming that large particles are less oblate than small ones. When the particle size increases, $Z_{h \hbar}$ increases, but because the particle has become less oblate, $Z_{d r}$ and $L_{d r}$ decrease. In [7], four stages in the melting process are distinguished. In the first stage small drops are formed at the tips of the ice branches, with melting occurring on the entire periphery, but mainly at the bottom of the snowflake. In the second stage the water is sucked into the inside of the snowflake, where it is accumulated. In the third stage the branches inside the snowflake start to melt, and consequently the structure of the snowflake is changed. In the fourth stage the snowflake collapses into a water drop. Because melting is most intense at the lower side of the snowflake, the "electromagnetic" shape of the particle becomes oblate, because the permittivity of water is much larger than the permittivity of the remaining unmelted snowflake. The negative correlation in the upper half of the melting layer can be understood by considering the melting process at a given height at the top of the layer more closely. All snowflakes are melting, but small snowflakes will melt sooner than large ones. The small particles will become oblate whereas large snowflakes still conserve their irregular shape. The ensembleaveraged shape of large particles is more or less spherical, implying that small melting snowflakes are on average more oblate than large ones, and so $Z_{h h}$ is negatively correlated to $Z_{d r}$ and $L_{d r}$. As melting proceeds, small snowflakes will turn into more or less spherical raindrops, and large snowflakes are now becoming oblate. Consequently, large particles are more oblate than small ones, and $Z_{h h}$ is positively correlated to $Z_{d r}$ and $L_{d r}$.

The density of a snowflake depends on its size. It is likely that the shape of the snowflakes is also related to the density; it is well known that loose snowflakes are irregularly structured, while ice crystals, having a high density, can be shaped like needles or plates. The measurements indicate that, for a fixed height, an increase of $Z_{h h}$ is caused by particles that are less oblate, because $Z_{d r}$ decreases. Combined radar and aircraft measurements in the melting layer indicated that wetted ice needles are often present just above the melting layer, and that a more pronounced $Z_{d r}$ is observed during very low reflectivities [8]. The cross-correlation analysis of this section shows that the phenomenon is more general: also during events with a strong bright band $Z_{d r}$ increases when $Z_{h h}$ decreases.

Fig. 4 shows the height profile of the cross-correlation coefficients of the radar observables, measured during the event of Fig. 2. In the upper half of the melting layer $Z_{h h}$ is negatively correlated to $Z_{d r}$ and $L_{d r}$. However, only in case of $Z_{h h}$ and $Z_{d r}$ a reversal is observed in the lower half of the melting layer. In case of $Z_{h h}$ and $L_{d r}$ the correlation remains negative, although it becomes smaller. The correlation coefficients are small, and only reveal tendencies. In the rain region too little data satisfies the minimum requirement of signal-to-noise ratio for accurate measurements; the calculations become unreliable.

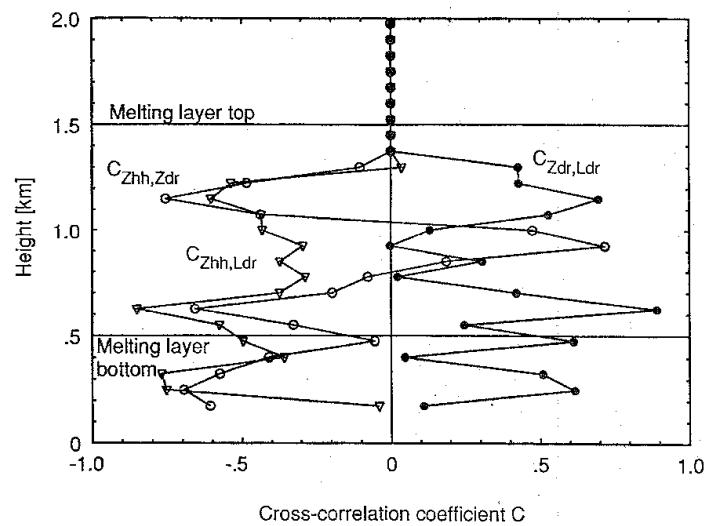

Fig. 4. Height profile of the cross-correlation coefficients of the polarimetric radar observables, during the event of Fig. 2 . The individual observables were integrated over $300 \mathrm{~m}$ and $32 \mathrm{~s}$. The correlation coefficients were calculated with $32 \mathrm{~min}$ worth of data.

\section{OUTLINE OF THE MODEL}

The model is a development of an earlier model [9]. Two new elements are introduced: the shape and the orientation of the melting snowflakes. A detailed description of the physics of the new melting layer model is given in [4] and [10]. In this paper only the salient points are described. Several assumptions are made.

- Each snowflake melts into one raindrop.

- The mass flux is constant through the melting layer.

- A monodisperse dropsize distribution suffices for modeling purposes.

- Melting snowflakes can be modeled as spheroids.

- A simple one-to-one relationship between size and shape is sufficient.

- The width of the orientation distribution of the particles decreases while the particles are melting.

Justification of these assumptions will be given when appropriate. The model is driven by two input parameters: the rain intensity below the melting layer and the mass density of the dry snowflakes above the melting layer. The latter is usually unknown, but necessary to explain specific radar observations.

\section{A. The Thickness of the Melting Layer}

The depth $h$ that the snowflake has descended in the melting layer is related to the melted mass fraction $f_{m}$ [3], [7]. This relationship is approximately given by

$$
f_{m}=\frac{1}{2}\left\{\sin \left(\frac{h}{H_{\max }} \pi-\frac{\pi}{2}\right)+1\right\} .
$$

$H_{\max }$ is the distance at which the snowflake is completely melted, and can be considered as the thickness of the melting layer; the top of the melting layer is located at $h=0$. The thickness of the melting layer is statistically related to the radar reflectivity of the rain just underneath it [3]. In [3], the second derivative of the height-dependence of $Z_{h h}$ is used to define the top of the melting layer, but comparison with 
a more extensive data set has shown that to result in an underestimation of the thickness with a factor 2 . Correcting it gives

$$
H_{\max }=200 Z_{\text {rain, } h h}^{0.17}
$$

with $H_{\max }$ given in meters and $Z_{\mathrm{rain}, h h}$ given in $\mathrm{mm}^{6} \mathrm{~m}^{-3}$. $Z_{\text {rain }, h h}$ is measured just below the melting layer. The data set is acquired during various weather conditions and probably encompasses different thermodynamic situations, assuming the relationship that is widely used to convert radar measurements during stratiform events into rain intensity $R$, e.g., [11]

$$
Z=200 R^{1.6}
$$

$H_{\max }$ is written as

$$
H_{\max }=492 R^{0.272}
$$

with $R$ in $\mathrm{mm} \cdot \mathrm{h}^{-1}$ : the thickness follows directly from the rain intensity.

\section{B. The Particle Size and Concentration}

Break up and coalescence, as well as particle growth due to condensation, are ignored: according to [2] they only occur in a narrow isothermal layer at the top of the melting layer, and in [3] they are shown to have a minor impact on the overall behavior of simulated reflectivity profiles of the melting layer. The size of a melting snowflake is then closely related to its mass density $\rho_{m s}$ and the size $D_{\text {rain }}$ of the resulting raindrop:

$$
D_{m s}=\rho_{m s}^{-(1 / 3)} D_{\text {rain }}
$$

The particle size can be calculated at any stage of melting, provided the mass density of the particle is known. The mass density of the melting snowflake is a function of the melted fraction and of the initial mass density of the snowflake before melting. Consequently, only the latter is necessary to calculate the size of the melting particle. In case of dry aggregated snow, the relationship between the particle size and mass density is known [3]. However, in reality hydrometeor types other than aggregated snow, like graupel or wetted ice crystals, occur as well. In the model, therefore, the mass density is used as an independent input parameter.

A monodisperse size distribution is used. The effective diameter $D_{r}$ of the raindrops is defined as the diameter that a drop would have if it had the mean volume of a cluster raindrops with a Marshall-Palmer size distribution $N(D)$ [11]:

$$
N(D)=N_{o} \exp \left[\frac{-3.67 D}{D_{o}}\right]
$$

in which $N_{o}=8 \cdot 10^{6}\left[\mathrm{~m}^{-4}\right]$ and $D_{o}$ is the diameter of the median dropvolume, while

$$
D_{r}=\left(\frac{V_{t}}{N_{t}}\right)^{1 / 3}
$$

with $V_{t}$ and $N_{t}$ as, respectively, the total volume and the number concentration of the raindrops. This results in

$$
D_{r}=0.495 D_{o}
$$

which in case of the Marshall-Palmer dropsize distribution is easy to convert into the rain intensity since [11]

$$
D_{o}=\frac{3.67}{4.1} R^{0.21}
$$

As a consequence of the assumed stationarity, the number concentration $N_{m s}$ of melting particles is given by

$$
N_{m s}=\frac{v_{r}}{v_{m s}} N_{r}
$$

with $V_{m s}$ and $V_{r}$ as the fall speed of the melting particle and of the raindrop it eventually becomes. $N_{r}$ is the number concentration of raindrops. Note that the concentration decreases during melting, which is in agreement with the experimental results given in [2].

\section{The Fall Speed of Melting Snowflakes}

The fall speed of melting snowflakes is obtained from balaning the gravitational forces, the drag forces, and the buoyancy of the snowflake [12],

$$
v_{m s}=\sqrt{\frac{4 g D_{m s} \rho_{m s}}{3 \rho_{a} C_{d}}}
$$

in which $g$ is the acceleration of gravity, $\rho_{a}$ as the mass density. of air, and $C_{d}$ the drag coefficient. The equation is derived for spherical particles. Spheroidal particles may have a somewhat different fall speed, because $C_{d}$ depends on the shape of the particle, but in this study no attempt is made to model it. For dry snowflakes $C_{d}$ equals approximately 1.2 , but for melting snowflakes it shows a large scatter between 0.6-1.2 [12]. Unfortunately, little is known about the relationship between $C_{d}$ and the melting process. Following the approach of [12], the drag coefficient of melting snowflakes is obtained through a linear interpolation between the $C_{d}$ values of the initial snowflake and the resulting raindrop,

$$
C_{d}=\frac{1.2-C_{d r}}{D_{s}-D_{r}}\left(D_{m s}-D_{s}\right)+1.2
$$

in which $C_{d r}$ is the drag coefficient of the raindrop, $D_{s}$ the diameter of the initial snowflake, and $D_{r}$ the diameter of the raindrop. $C_{d r}$ is obtained from (with $\rho_{r}$ as the mass density of water)

$$
C_{d r}=\frac{4 g D_{r} \rho_{w}}{3 \rho_{a} v_{r}^{2}}
$$

in which the fall speed $v_{r}$ of the raindrop is calculated from the well-known relationship [13]

$$
v_{r}=9.65-10.3 e^{-0.6 D_{r}} .
$$

Note that the fall velocity of melting snowflakes, due to its dependence upon $D_{r}$, is uniquely related to the rain intensity. In the model, $\rho_{a}$ is set to $1.3 \cdot 10^{-3} \mathrm{~g} \cdot \mathrm{cm}^{-3}$.

The mean Doppler velocity $V_{d m s}$ of a melting snowflake is the mean, power-weighted, fall velocity. The equivalent 
reflectivity factor $Z_{m s}$ of melting snow is by definition related to the radar cross-section $\sigma_{m s}^{\text {tot }}$ as

$$
\begin{aligned}
Z_{m s} & =\frac{\lambda^{4}}{\left|K_{r}\right|^{2} \pi^{5}} \sigma_{m s}^{\text {tot }} \\
& =\frac{\lambda^{4}}{\left|K_{r}\right|^{2} \pi^{5}} \frac{\left|K_{m s}\right|^{2} \pi^{5}}{\lambda^{4}} \int N_{m s}\left(D_{m s}\right) D_{m s}^{6} d D_{m s}
\end{aligned}
$$

and consequently $V_{d m s}$ can be related to the mean Doppler velocity $V_{d r}$ of rain:

$$
\begin{aligned}
V_{m s} & =\frac{1}{\sigma_{m s}^{\text {tot }}} \int v_{m s} N_{m s}\left(D_{m s}\right) D_{m s}^{6} d D_{m s} \frac{\left|K_{m s}\right|^{2} \pi^{5}}{\lambda^{4}} \\
& =\frac{1}{\sigma_{m s}^{\text {tot }}} \int v_{r} N_{r}\left(D_{r}\right) D_{r}^{6} d D_{r} \rho_{m s}^{-(7 / 3)} \frac{\left|K_{m s}\right|^{2} \pi^{5}}{\lambda^{4}} \\
& =\frac{\left|K_{m s}\right|^{2}}{\left|K_{r}\right|^{2}} \frac{Z_{r}}{Z_{m s}} \rho_{m s}^{-(7 / 3)} V_{d r} .
\end{aligned}
$$

The mean Doppler velocity depends on the mass density of the snowflakes, and on the permittivity. It implicitly has been assumed that the mass density of melting snowflakes is independent of particle size.

\section{The Shape of Melting Snowflakes}

Most water is found at the bottom of the melting snowflakes, forming a lens-like shape [14]. The permittivity of water is much larger than that of the ice in the particle, which suggests to model the "electromagnetic" shape of the particle as a spheroid; the shape changes during melting, because the water content changes. The correlation analysis of Section $\mathrm{V}$ suggests that small melting snowflakes appear as more oblate than large ones in the upper half of the melting layer; in the lower half of the melting layer large snowflakes appear as more oblate than small ones. Therefore, the axial ratio of snowflakes is modeled as a function of size, with the slope of this function changing from positive to negative during melting. For reasons of simplicity, and considering that the size-shape relationship is probably highly stochastic, a linear function between the axial ratio $\xi_{s}$ and the snowflake diameter $D_{s}$ is proposed:

$$
\begin{aligned}
\xi_{s}\left(D_{s}\right)= & \xi_{1}+\frac{\xi_{2}-\xi_{1}}{D_{\max }-D_{\min }} \\
& \cdot D_{s}-\frac{\xi_{2}-\xi_{1}}{D_{\max }-D_{\min }} D_{\min }
\end{aligned}
$$

with $\xi_{1}$ and $\xi_{2}$ as the axial ratios, respectively, of the smallest and largest particle with diameters $D_{\min }$ and $D_{\max }$. At the onset of melting, $\xi_{1}$ is smaller than $\xi_{2}$, but during melting both parameters change. For dry snow $\xi_{2}$ is set to 0.9 , which approximates the mean result given in [15]; in the next section $\xi_{1}$ will be determined from comparison of model calculations with radar data.

The model uses the effective dropsize based on the Marshall-Palmer distribution. The axial ratio should therefore also be related to an effective dropsize. For modeling of radar measurements, the reflectivity-weighted effective axial ratio is used. However, propagation phenomena like attenuation approximately depend on the volume of particles, which suggests to use the volume-weighted axial ratio to model the propagation through the melting layer. The effective axial ratios are obtained from

$$
\xi_{\mathrm{eff}}^{\mathrm{rad}, \operatorname{prop}}\left(D_{r}\right)=\frac{\int \xi_{m s}(D) N\left(D, D_{r}\right) D^{\alpha} d D}{\int N\left(D, D_{r}\right) D^{\alpha} d D}
$$

with $\alpha=3$ in case of volume-weighting and $\alpha=6$ in case of reflectivity-weighting. The superscripts rad and prop refer to the radar and propagation case, respectively. After melting the Morrison-Cross relationship [16] of the axial ratio $\xi^{\text {rain }}(D)$ of raindrops is used:

$$
\xi^{\text {rain }}(D)=1.0-0.05 D \quad[D \text { in } \mathrm{mm}]
$$

The effective axial ratios are then given by

$$
\begin{aligned}
\xi_{r}^{\mathrm{rad}} & =1.0-0.19 D_{r} \\
\xi_{r}^{\mathrm{prop}} & =1.0-0.11 D_{r}
\end{aligned}
$$

with $D_{r}$ in mm. The shape of a melting snowflake results from internal, e.g., capillary, forces as well as external forces, like gravity, drag, and buoyancy. It depends on both the mass and volume of the particle. However, it is intuitively felt that the shape of a melting snowflake is more strongly related to its volume than to its mass, because the mass does not change, whereas the volume and shape do. An optimum fit of model simulations to radar data, presented in Section VIII, is obtained when

$$
\xi_{m s}=\xi_{s}+\left(\xi_{r}-\xi_{s}\right) f_{v}^{8}
$$

in which $f_{v}$ is the melted volume fraction of the particle.

\section{E. The Orientation of Melting Snowflakes}

A melting snowflake is modeled like a spheroid with its orientation-angle defined by its axis of rotational symmetry. However, a melting snowflake is a complex particle, of which it is difficult to speak of rotational symmetry. As will appear, the modeled orientation-angle may also represent effects due to the irregular shape and motion of the particle.

Raindrop canting is caused by wind shear and turbulence. It is likely that the same holds for melting snowflakes. But there is more, as described in [7]. Before melting the motion of a snowflake can be correlated to the shape, size, and mass. However, as soon as melting starts, the particle starts to move in a completely random order, independent of shape, size, and mass: helical loci are interrupted by linear movements, swinging, and rotation. This lasts until the ice lattice of the particle collapses, from which moment on the particle starts to accelerate in a downwards motion. No tumbling is observed. The observations were done in a wind tunnel with constant air stream and temperature gradient, and so no external forces 
would have caused this random motion. Probably internal forces, caused by a nonuniform distribution of melt water inside the snowflake, force the particle into this behavior.

Polarimetric radar measurements are sensitive to the shape and orientation of the particles. The apparent shape and orientation of the particles is defined by the projection of the geometry of the particle on the plane of incidence of the radar wave. Radar measurements can always be related to the apparent shape and orientation. Suppose that the irregularlyshaped melting snowflakes are moving in some random order, then the radar signals they cause can be related to apparent particles with a varying apparent shape and orientation in the plane of incidence of the radar wave. In the model the distribution of orientation angles is used to account for the effect of particle motion. A uniform distribution of the solid angle $\Omega$ of the symmetry-axes of the particles is assumed. The solid angle is specified by the azimuth angle $\alpha$ and the elevation angle $\delta$ (relative to the vertical). The azimuth angle of the axis of symmetry of the snowflake is assumed to be uniformly distributed between $0-2 \pi$. The sine of the elevation angle (defined relative to the vertical) is also uniformly distributed, up to a certain (variable) width. Summarizing:

$$
\begin{aligned}
\int p(\Omega) d \Omega= & \frac{1}{2 \pi} \frac{1}{1-\cos \Delta \delta_{m s}} \\
& \cdot \int_{0}^{2 \pi}\left\{\int_{0}^{\Delta \delta_{m s}} \sin \delta d \delta\right\} d \alpha=1
\end{aligned}
$$

in which $\Delta \delta_{m s}$ is the maximum elevation angle. Since the randomness of particle motion decreases toward the end of melting, the width of the orientation distribution decreases as well. An optimum fit of model simulations to radar data, as shown in Section VIII, is obtained when

$$
\Delta \delta_{m s}=\Delta \delta_{s}+\left(\Delta \delta_{r}-\Delta \delta_{s}\right) f_{v}^{3}
$$

in which $\Delta \delta_{s}$ and $\Delta \delta_{r}$ are the widths corresponding to the orientation of, respectively, unmelted snow and rain.

\section{F. The Permittivity of Melting Snowflakes}

It is shown in [17] that accurate knowledge of the permittivity is necessary, because the calculated propagation profiles depend strongly on it. A mixing formula that is able to calculate the permittivity of melting snow is the Maxwell Garnet theory, that treats a mixture as a matrix of homogeneous material that surrounds inclusions of an other material. In the melting layer model, the formulation of [18] is used. The Maxwell Garnet theory deals with a two-component mixture. In case of melting snow, which consists of three components, the mixing rules must be applied twice. The use of mixing formulas requires some knowledge of the structure of the melting snowflakes; what constituent should be taken as the matrix, and what as the inclusion? Observation of the melting process [7] reveals 1) that melting occurs at the periphery of the snowflake and 2) that melt water is sucked inside to form a film around the ice branches. The first

\begin{tabular}{|c|c|c|c|}
\hline \multicolumn{2}{|c|}{ Model parameters } & \multicolumn{2}{|l|}{ Equipment parameters } \\
\hline$\xi_{1}$ & 0.3 & Satellite frequency [GHz] & 30 \\
\hline$\xi_{2}$ & 0.9 & Radar frequency [GHz] & 3 \\
\hline$\Delta \delta_{\mathrm{s}}$ & $70^{\circ}$ & Antenna elevation & $30^{\circ}$ \\
\hline$\Delta \delta_{\mathrm{r}}$ & $55^{\circ}$ & Polarization angle & $20^{\circ}$ \\
\hline$\rho_{\mathrm{s}}\left[\mathrm{gcm}^{-3}\right]$ & 0.1 & Rain intensity $\left[\mathrm{mmh}^{-1}\right]$ & 5 \\
\hline
\end{tabular}

TABLE II

Parameters for Simulation of Radar and Propagation MEASUREMENTS

phenomenon suggests using water as matrix around snow, the second one suggests using air in a matrix of wet ice. In the model both options are calculated and, in the end, the average of the results from the two methods is used.

\section{SimUlations OF RADAR MEASUREMENTS}

Some typical examples of the backscattering by and propagation through the melting layer will be discussed. The experimental set up of the, earlier mentioned, Olympus measurement campaign at Delft University is used as basis for the simulations. Table II gives the model and equipment parameters. The antenna elevation angle is defined with respect to the horizontal. The polarization angle is defined in the plane of incidence of the radar waves as the angle between the $E$-vector and the horizontal. The mass density of the dry snowflakes is denoted by $\rho_{s}$. The given model parameters are obtained by a first order tuning of the model to the radar observations that were discussed in Section IV.

\section{A. Height Profiles}

Fig. 5(a)-(c) give height profiles of the radar as well as propagation observables. The radar observables follow the typically observed trends of Section IV. The $C P A, X P D$, and $C P D$ exhibit a peak in the lower half of the melting layer. The peak of $C P A$ coincides with the peak of $Z_{d r}$, but the $X P D$ and $C P D$ peaks are slightly shifted downwards. The phase patterns are different: $\phi_{h h}$ and $\phi_{h h}-\phi_{v v}$ exhibit a peak, but $\phi_{h h}-\phi_{h v}$ does not. The antenna elevation angle is set to $30^{\circ}$, implying that half of the value of $V_{d}$ that would be obtained with a zenith-pointing radar is calculated.

In the following subsections the effect of model parameters on the height profiles of the radar observables is given. The effect on the propagation observables is not given, because they appear as range-integrated parameters in the performance of satellite links; they will discussed in Section IX.

1) Effect of the Mass Density: In the model, the size of a snowflake is determined by its mass density and the diameter it would have after melting. The larger the mass density, the smaller and consequently the more oblate the particle is. Fig. 6(a) and (b) give the height profiles of the radar observables, calculated with $\rho_{s}=0.05,0.1$, and $0.5 \mathrm{~g} \cdot \mathrm{cm}^{-3}$. The remaining input parameters are given in Table $\mathrm{I}$.

When the mass density of the initial snowflakes increases $Z_{h h}$ decreases, because the particles become smaller. Note that the strength of the bright band decreases rapidly for high mass densities. The $Z_{d r}$ and $L_{d r}$ profiles exhibit a peak in all three cases. These, colocated, peaks shift upwards, broaden and increase when the mass density increases. The simulated 


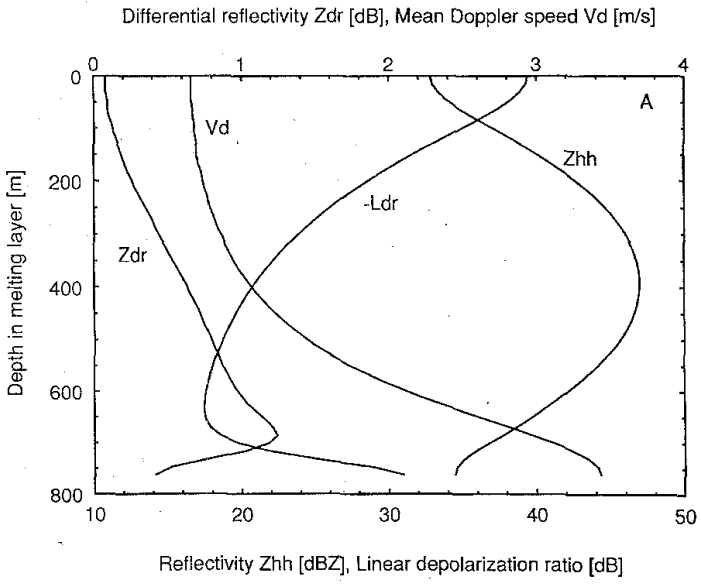

(a)

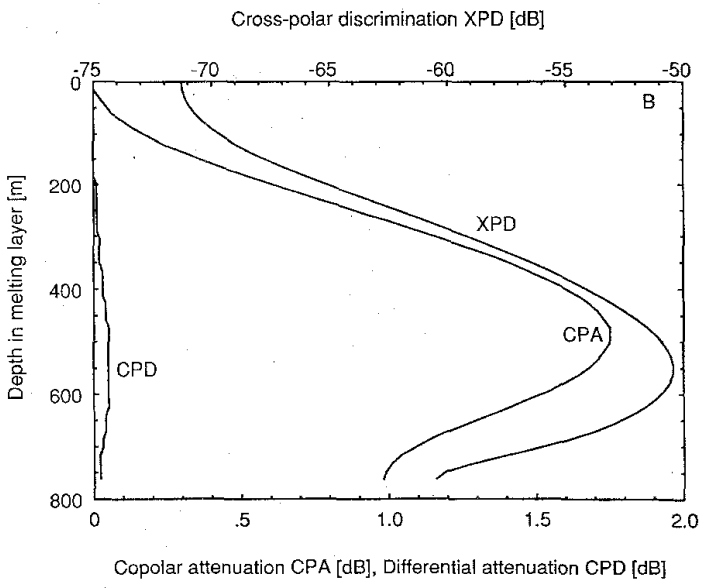

(b)

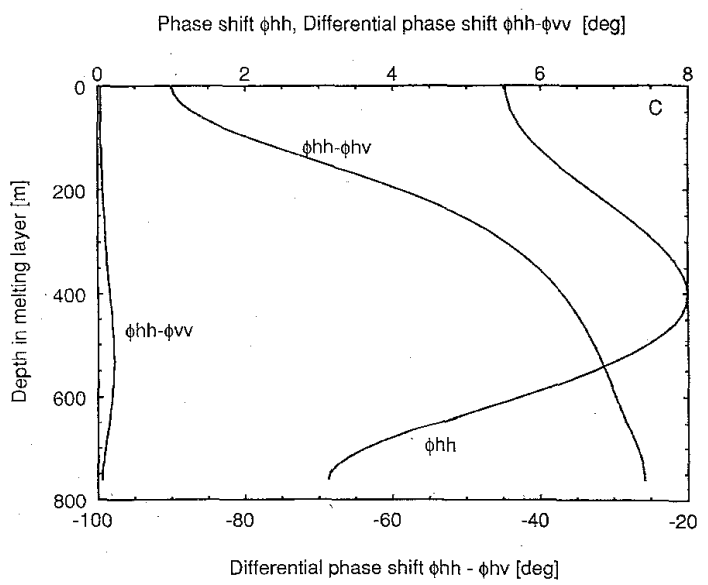

(c)

Fig. 5. A typical example of the radar and propagation observables in the melting layer, calculated with the parameters of Table II: (a) gives the radar observables; (b) and (c) give the propagation parameters.

trend agrees with the observation of Section III. The fall speed $V_{d}$. increases monotonically during melting. The velocity at

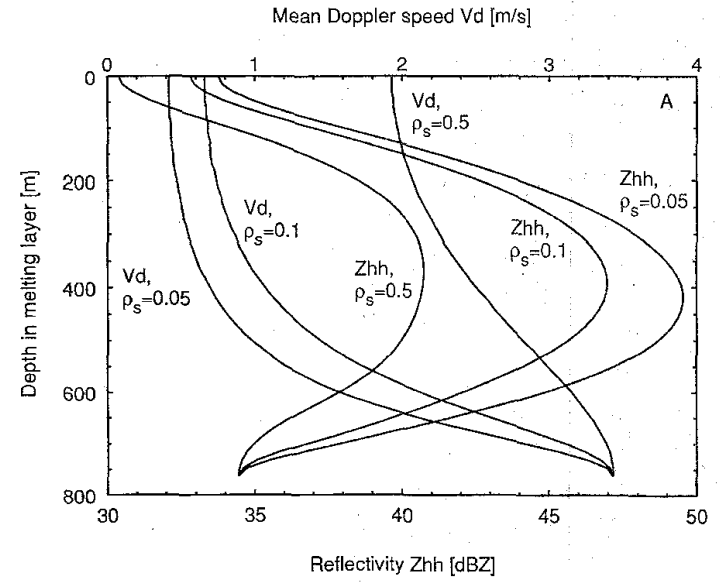

(a)

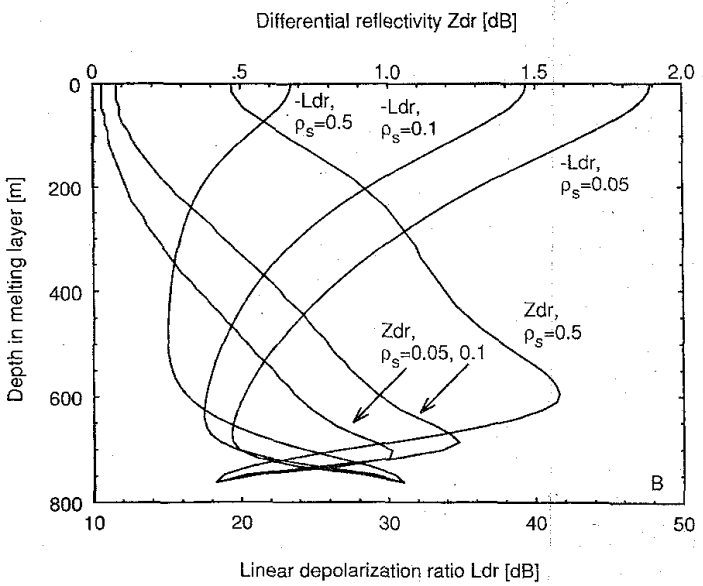

(b)

Fig. 6. Effect of the initial mass density on the radar observables in the melting layer, calculated with the parameters of Table II.

the top of the melting layer increases when the mass density increases, since the particles encounter less air resistance.

2) Effect of the Axial Ratio: The effect of the axial ratio was investigated by setting the axial ratio $\xi_{1}$ of the smallest snowflake to $0.3,0.6$, and 0.9 , and $\xi_{2}$ to 0.9 . The results are shown in Fig. 7(a) and (b). $Z_{h h}$ is hardly dependent upon the axial ratio: it varies less than $1 \mathrm{~dB}$ when $\xi_{1}$ varies. The $Z_{d r}$ and $L_{d r}$ profiles, however, change significantly. When $\xi_{1}=0.9$, implying more or less spherical snowflakes, no peak is observed at all. For $\xi_{1}<0.9$ the peak appears, and increases when $\xi_{1}$ decreases. The $Z_{d r}$ and $L_{d r}$ peaks coincide and do not shift up or down when the axial ratio is varied. The simulated fall speed does not depend on the axial ratio, but is given here for the sake of completeness. The real fall speed may, however, depend on the particle shape, but the model does not take this into account.

3) Effect of the Particle Orientation: The effect of the particle orientation is varied by setting the spread $\Delta \delta_{s}$ of the orientation distribution of dry snowflakes to $90^{\circ}, 70^{\circ}$, and $55^{\circ}$, and keeping $\Delta \delta_{r}$ at $55^{\circ}$ for the resulting raindrops. The results are shown in Fig. 8(a) and (b). $Z_{h h}$ is hardly affected by the 


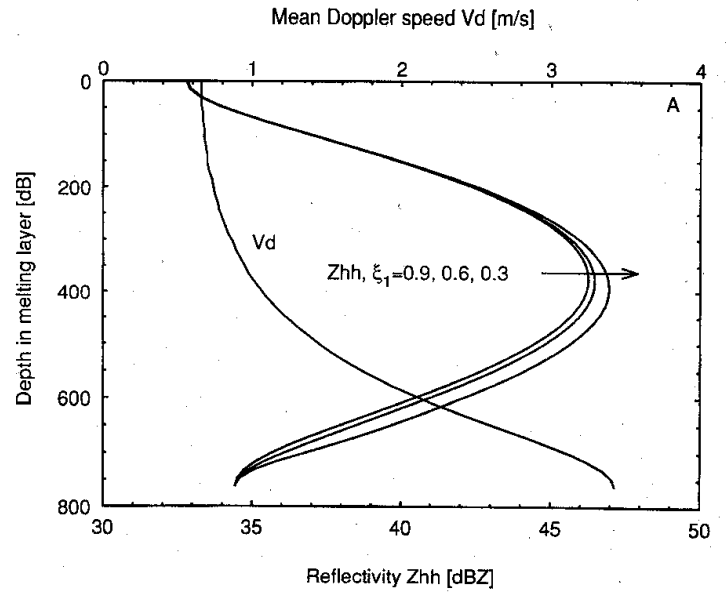

(a)

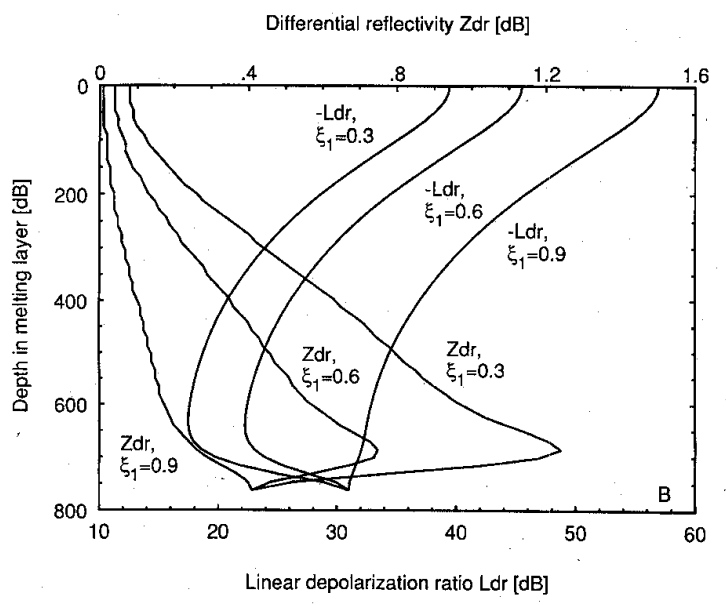

(b)

Fig. 7. Effect of the initial particle shape on the radar observables in the melting layer, calculated with the parameters of Table II.

orientation of the snowflakes: it varies a few tenths of a decibel when $\Delta \delta_{s}$ varies. $L_{d r}$ increases when the particle canting increases, but $Z_{d r}$ decreases then. $Z_{d r}$ is more sensitive to canting variations than $L_{d r}$; the increase of $L_{d r}$ is small compared to the decrease of $Z_{d r}$. Changing the orientation significantly affects the location of the peaks. The $Z_{d r}$ peak moves downwards when the particle canting increases, but the location of the $L_{d r}$ peak does not change: the two peaks no longer coincide. The orientation angle was defined to account for the irregular motions of melting snowflakes; it does not necessarily represent real particle canting. However, the concept describes the typically observed behavior of $Z_{d r}$ and $L_{d r}$ well.

\section{COMPARISON WITH RADAR MEASUREMENTS}

Radar measurements with the S-band Chilbolton radar of the Rutherford Appleton Laboratory in the U.K. have been used to test the model. The Chilbolton radar measures $Z_{d r}$ and $L_{d r}$ during two separate vertical scans. During each scan the reflectivity is measured. Figs. 9 and 10 show the

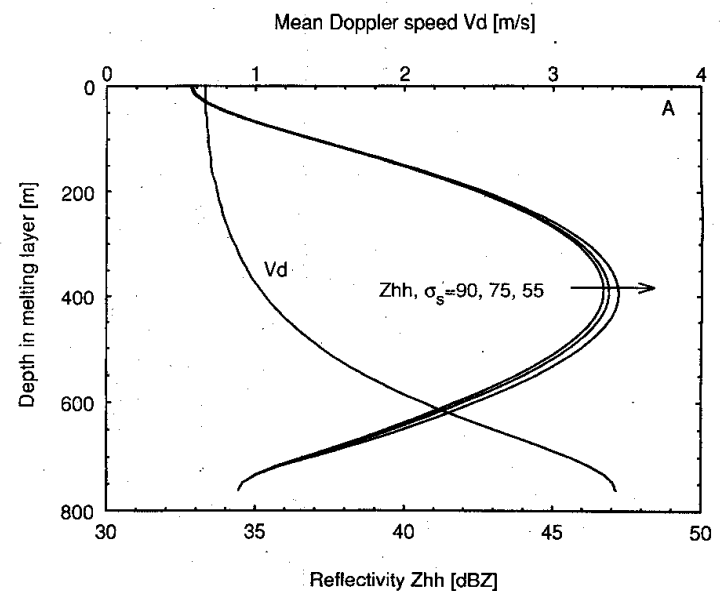

(a)

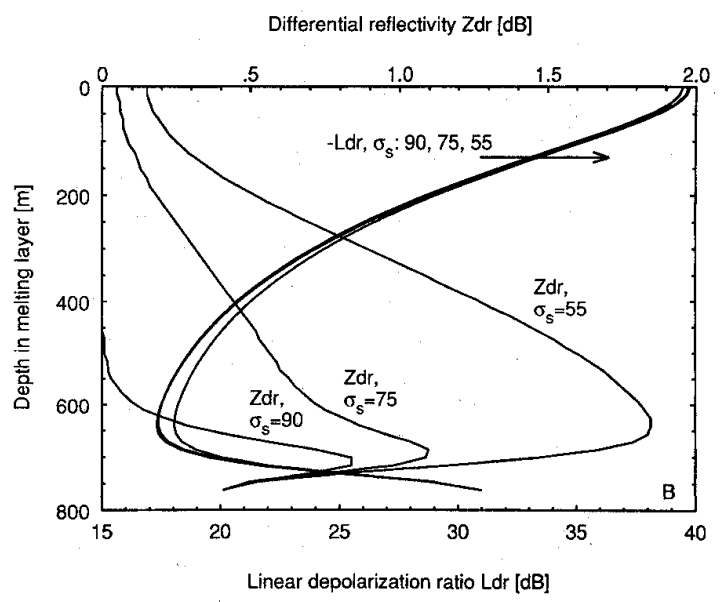

(b)

Fig. 8. Effect of the initial particle orientation on the radar observables in the melting layer, calculated with the parameters of Table II.

Chilbolton data and model comparisons for two different situations; in each plot both scans of the reflectivity are given. The measurement of Fig. 9 is simulated with an rain intensity of $2 \mathrm{~mm} \cdot \mathrm{h}^{-1}$ and an initial mass density of the snowflakes of $0.15 \mathrm{~g} \cdot \mathrm{cm}^{-3}$. In Fig. 10, the rain intensity is set to 1.5 $\mathrm{mm} \cdot \mathrm{h}^{-1}$ and the mass density to $0.3 \mathrm{~g} \cdot \mathrm{cm}^{-3}$. The radar observations and model simulations are in good agreement, in both cases. Not all analyzed data, however, could be simulated by the model as it is, but slightly changing (42) $\left(\Delta \delta_{r}\right.$ had to be varied over a range of $10^{\circ}$ ) resulted in a good fit again. Apparently, the physics that are fundamental to the melting layer are adequately described, although the specific parameters of the model may need some adjustment to predict all measurements in detail. However, it is surprising that such a complex phenomenon like the melting layer can be described by a model with only a few variables.

\section{APPLICATION TO THE Propagation of SATEllite Signals}

Satellite receivers experience the overall effect of the melting layer. Fig. 11 gives the excess propagation observables 


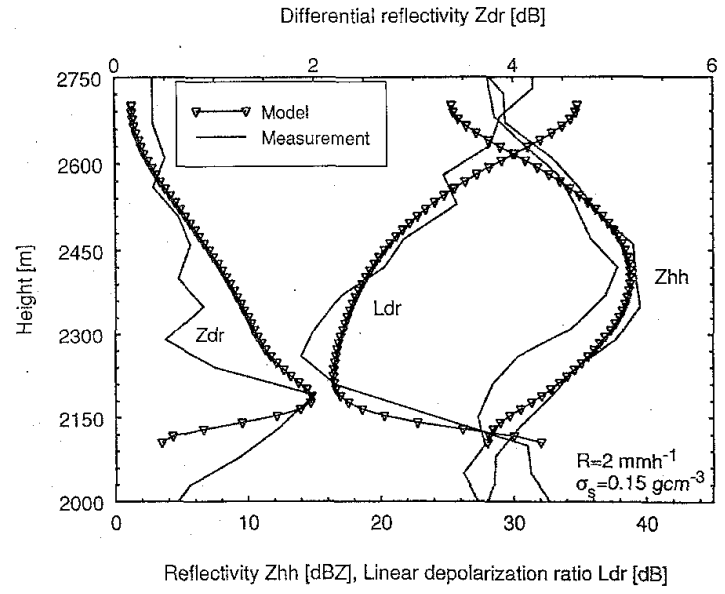

Fig. 9. Comparison of model sinulations and a radar measurement with the Chilbolton radar. Details in Table I. The $Z_{d r}$ and $L_{d r}$ are simultaneously measured with $Z_{h h}$ during different vertical scans.

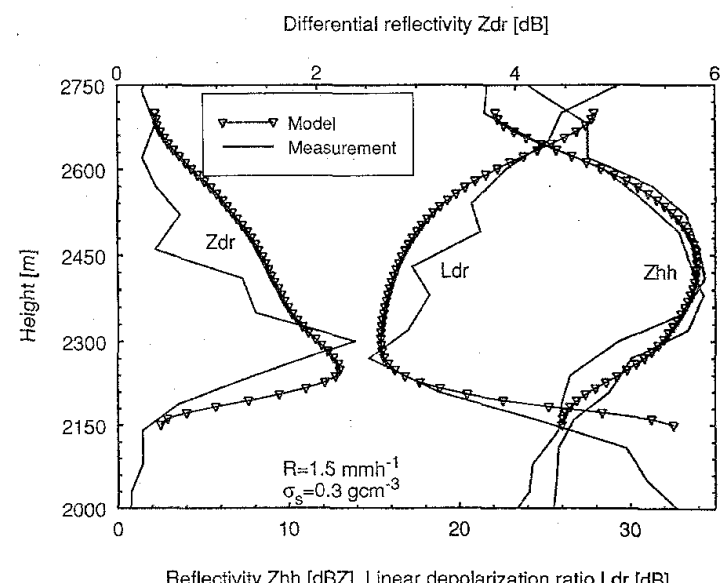

Fig. 10. Comparison of model simulations and a radar measurement with the Chilbolton radar. Details in Table 1 . The $Z_{d r}$ and $L_{d r}$ are simultaneously measured with $Z_{h h}$ during different vertical scans.

integrated over the entire melting layer as function of the rain intensity just underneath it, calculated with the set up of Table II. In a real life situation, effects of the rain below and the ice crystals above the melting layer should be taken into account. $e C P A$ increases almost linearly with the rain intensity, and becomes approximately $2 \mathrm{~dB}$ at $20 \mathrm{~mm} / \mathrm{h}$. The predicted values are in good agreement with the calculations of [19], which are based on the Mie theory, whereas the present model uses the extended Rayleigh approximation. $e C P D$ is very small: less than $0.2 \mathrm{~dB}$. eXPD decreases with rain intensity. It is very large at low rain intensities, and decreases toward $6 \mathrm{~dB}$ at $20 \mathrm{~mm} \cdot \mathrm{h}^{-1}$. $e \phi_{h h}$ increases with rain intensity: at $20 \mathrm{~mm} / \mathrm{h}$, $e \phi_{h h}=40^{\circ}$, and $e\left(\phi_{h h}-\phi_{v v}\right)=1.5^{\circ} \cdot e\left(\phi_{h h}-\phi_{h v}\right)$ and $e\left(\phi_{h h}-\phi_{v v}\right)$ do not vary significantly. Most rain events with a bright band will have a rain intensity lower than $10 \mathrm{~mm} \cdot \mathrm{h}^{-1}$. Keeping that in mind, it can be concluded that at $30 \mathrm{GHz}$, the additional effect of the melting layer is significant only for the cross-polar discrimination, the copolar phase shift, and the cross-polar differential phase shift. For the other propagation

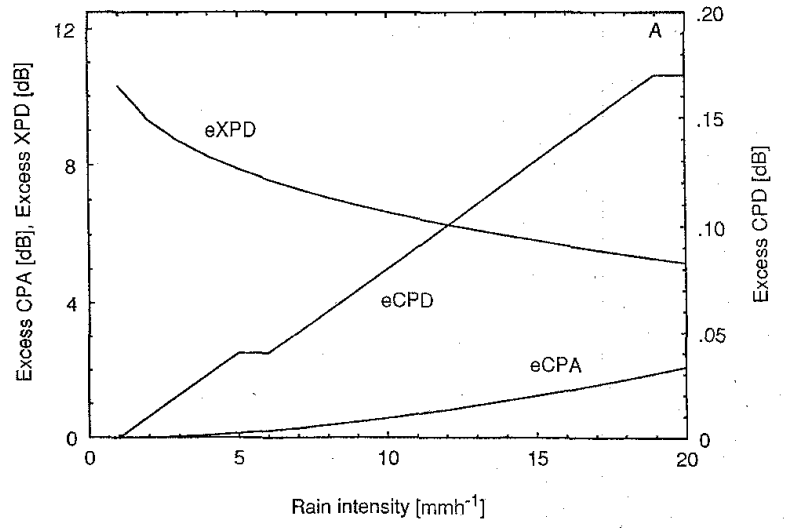

(a)

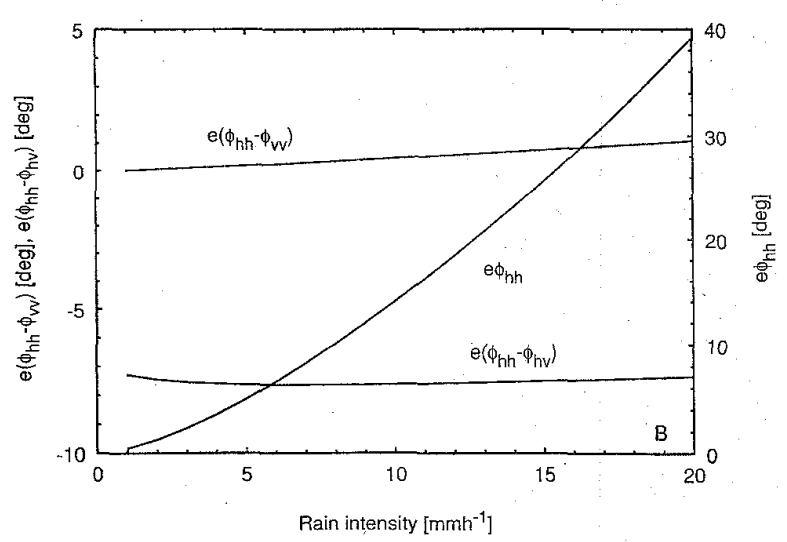

(b)

Fig. 11. Range-integrated excess propagation observables as function of the rain intensity, calculated with the parameters of Table II.

parameters, the melting layer can be treated as if it were a layer of rain.

Fig. 12 show the excess propagation observables integrated over the whole melting layer as function of the frequency, again calculated with the set up of Table $\Pi$. eCPA increases until the frequency is approximately $20 \mathrm{GHz}$. At $35 \mathrm{GHz}$, it becomes negative, meaning that the influence of the melting layer is less than the influence of a similar layer of rain. At high frequencies, the influence of the melting layer should not be calculated by replacing it with rain, but should to some extent be discarded. $e C P D$ does not vary much with the frequency. eXPD decreases with frequency, but at low frequencies the melting layer has a large impact on the XPD of radio signals. Of the phase observables, only $e\left(\phi_{h h}-\phi_{h v}\right)$ and $e \phi_{h h}$ change significantly with frequency.

In Fig. 13 the excess propagation observables are given as a function of the mass density of the snowflakes before melting. Only for densities smaller than $0.2 \mathrm{~g} \cdot \mathrm{cm}^{-3}$ do the observables vary significantly. Most of the radar measurements are simulated with a mass density larger than $0.1 \mathrm{~g} \cdot \mathrm{cm}^{-3}$ : the model predicts that the additional effect of the melting layer can be neglected and even that the melting layer should, to some extend, be discarded. The melting layer may, however, 


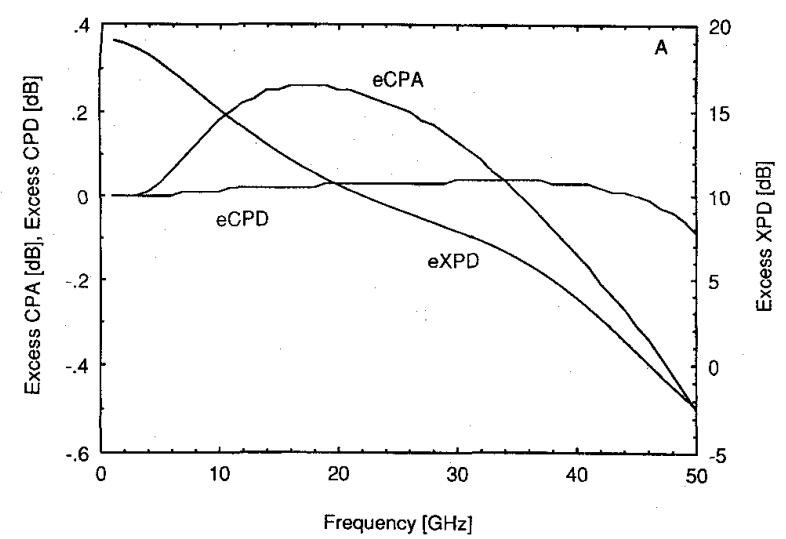

(a)

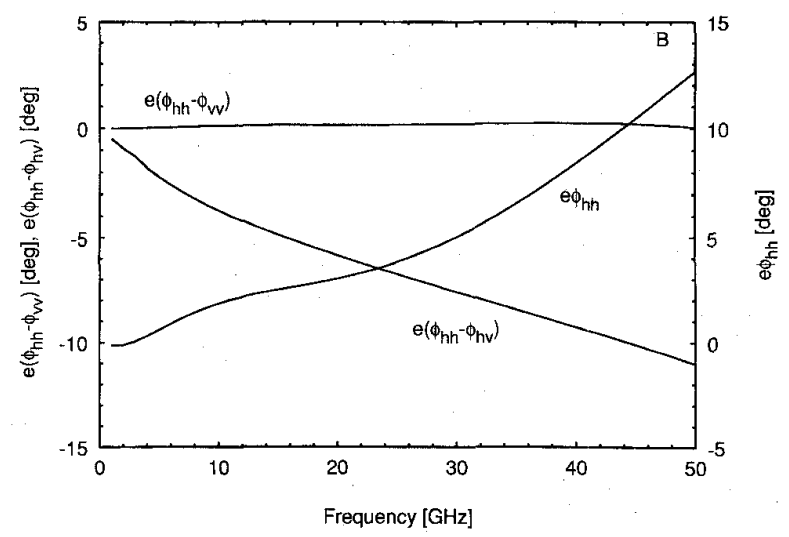

(b)

Fig. 12. Range-integrated excess propagation observables as function of the satellite frequency, calculated with the parameters of Table II.

cause values for the $X P D$ that are $10 \mathrm{~dB}$ in excess to the values a similar layer of rain would give.

\section{CONCLUSION}

A simple model of the melting layer is presented. It is able to predict Doppler and polarimetric radar measurements as well as propagation through the melting layer. The height profiles of the propagation and radar observables are similar: dependent upon the rain intensity and mass density of the snowflakes, they exhibit a peak somewhere in the melting layer. When the orientation of the melting snowflakes changes during melting, the peaks of $L_{d r}$ and $Z_{d r}$ do not coincide. The effect of the melting layer on radio signals increases with increasing rain intensity.

The model is based on several assumptions regarding the change of particle shape and orientation during melting. These assumptions are based on only a few typical radar measurements. Comparison of the model with a more extensive data set from radar as well as propagation experiments may lead to different values of the used parameters.

The model requires two input parameters: the rain intensity and the mass density of the snowflakes before they melt. The latter is usually not available, and when it is small it appears to have a significant effect. However, because the model is

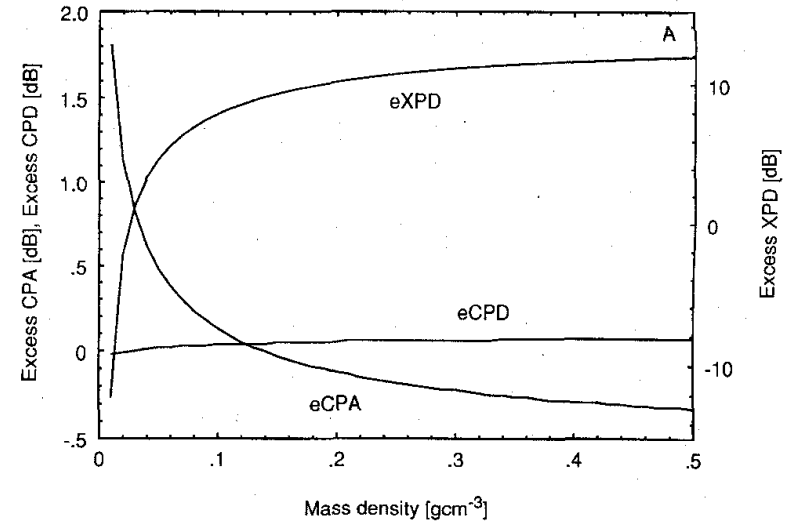

(a)

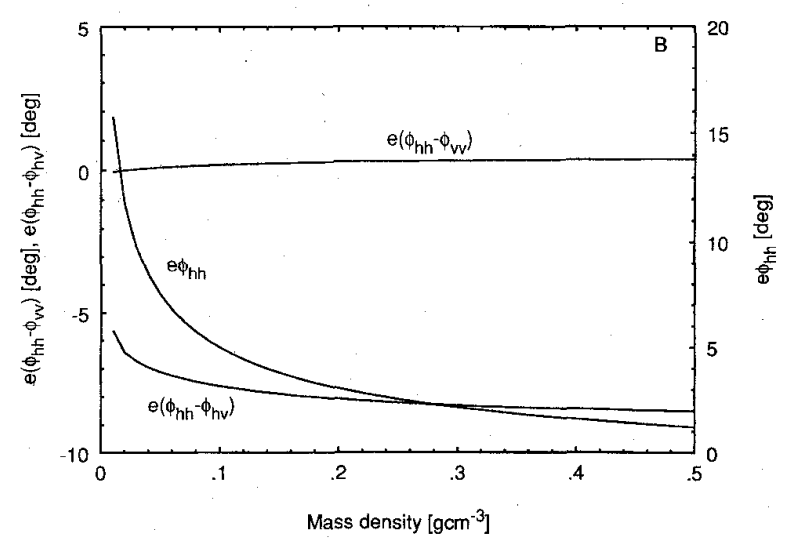

(b)

Fig. 13. Range-integrated excess propagation observables as function of the mass density, calculated with the parameters of Table II.

based on one effective particle with average properties, it is expected that small values of the mass density do not apply; radar reflectivity measurements can be simulated with a value of the mass density larger than $0.1 \mathrm{~g} / \mathrm{cm}^{3}$.

\section{ACKNOWLEDGMENT}

The authors would like to thank J. W. F. Goddard of Rutherford Appleton Laboratories for his kindness to provide radar data to test the model.

\section{REFERENCES}

[1] J. P. V. Poiares Baptista, Ed., OPEX Second Workshop; Reference Book on Radar, vol. 4. Noordwijk, The Netherlands: ESA-ESTEC, 1994 (WPP-083).

[2] P. T. Willis and A. J. Heymsfield, "Structure of the melting layer in mesoscale convective system stratiform precipitation," J. Atmos. Sci., vol. 46, pp. 2008-2025, 1989.

[3] W. Klaassen, "Radar observations and simulation of the melting layer of precipitation," J. Atmos. Sci., vol, 45, pp. 3741-3753, 1989.

[4] H. W. J. Russchenberg, Ground-Based Remote Sensing of Precipitation with a Multi-Polarized FM-CW Doppler Radar. Delft, The Netherlands: Delft Univ. Press, 1992 (ISBN 90-6275-823-1).

[5] D. A. De Wolf, H. W. J. Russchenberg, and L. P. Ligthart, "Lineof-sight propagation through rain at 5-90 GHz: Simplified analysis," in Proc. URSI Comm. F Open Symp. Wave Propagation and Remote Sensing, Ravenscar, UK, 1992, pp. 10.1.1-10.1.4.

[6] P. M. Austin and H. C. Bemis, "A quatitative study of the bright band in radar precipitation echoes," J. Appl. Met., vol. 7, pp. 145-151, 1950. 
[7] S. K. Mitra, O. Vohl, M. Ahr, and H. R. Pruppacher, "A wind tunnel and theoretical study of the melting behavior of atmospheric ice particle. IV: Experiment and theory for snow flakes," J. Atmos. Sci., vol. 47, pp. $585-591,1990$.

[8] P. Meischner, V. N. Bringi, M. Hagen, and H. Höller, "Multiparameter radar characterization of a melting layer compared with in situ measurements," in Proc. 25th Radar Meteorology Conf., Paris, 1991, pp. $721-724$.

[9] B. E. Ekpenyong and R. C. Srivastava, "Radar measurements of the melting layer-A theoretical study," in Proc. 14th Radar Meteorology Conf., AMS, Tucson, AZ, 1970, pp. 161-166.

[10] H. W. J. Russchenberg and L. P. Ligthart, "Backscattering by and propagation through the melting layer of precipitation," ESTEC Final Rep. PO 122859, Noordwijk, The Netherlands, 1993.

[11] L. J. Battan, Radar Observation of the Atmosphere. Chicago, IL: Univ. Press Chicago, 1973.

[12] T. Matsuo and Y. Sasyo, "Melting of snowfiakes below freezing level in the atmosphere," J. Met. Soc., Japan, vol. 59, no. 1, pp. 10-24, 1981.

[13] D. Atlas, R. C. Srivastava, and R. S. Sekhon, "Doppler and radar characteristics of precipitation at vertical incidence," Rev. Geophys. Space Phys., vol. 11, no. 1, pp. 1-35, 1973.

[14] Y. Fujiyoshi, "Melting snowflakes," J. Atmos. Sci., vol. 43, pp. 307-311, 1986.

[15] C. Magono and T. Nakamura, "Aerodynamic studies of falling snow flakes," J. Met. Soc., Japan, vol. 43, pp. 139-147, 1965.

[16] J. A. Morrison and M. J. Cross, "Scattering of a plane electromagnetic wave by axisymmetric raindrops," Bell Syst. Tech. J., vol. 53, pp. 955-1019, 1974.

[17] R. A. Hulays and M. M. Z. Kharadly, "Modeling of melting snow for scattering and attenuation calculations," in Proc. ICAP 1993 IEE Conf. Publ, 1993, no. 370, pp. 2873-2876.
[18] D. A. De Wolf, H. W. J. Russchenberg, and L. P. Ligthart, "Effective permittivity of and scattering from wet snow and ice droplets at weather radar wavelengths," IEEE Trans. Antennas Propagat., vol. 38, pp. $1317-1325,1990$.

[19] W. Klaassen, "Attenuation and reflection of radio waves by a melting layer of precipitation," Proc. Inst. Elect. Eng., vol. 137, pt. H, no. 1, pp. 39-44, 1990.

H. W. J. Russchenberg graduated in 1986 . He received the Ph.D. degree in 1992 from Delft University of Technology.

Since 1986 he has been working on atmospheric radar remote sensing, applied to radio wave propagation, although new applications like climatology and hydrology are becoming increasingly important. Currently, he is Head of the remote sensing sector of the International Research Centre of Telecommunication-Transmission and Radar IRCTR.

L. P. Ligthart (M'94-SM'95) graduated with distinction in 1969. He received the Ph.D. degree from the Delft University of Technology in 1985

Since 1969 he has been working with the Microwave Laboratory, Delft University, working on transmission line theory, antennas, propagation, and radar. In 1988, he accepted a Part-Time Professorship, focusing on radar. In 1992, he became a Full-Time Professor in microwave transmission, remote sensing technology, and radar. Currently, he is the Director of the International Research Centre of Telecommunication-Transmission and Radar IRCTR. 